\title{
Problemas associados ao binge drinking entre estudantes das capitais brasileiras
}

\author{
Problems associated with binge drinking among students \\ in Brazil's state capitals
}

\author{
Karla Gomes ${ }^{1}$ \\ Tatiana de Castro Amato ${ }^{1}$ \\ André Bedendo ${ }^{1}$ \\ Elaine Lucas dos Santos ${ }^{1}$ \\ Ana Regina Noto ${ }^{1}$
}

${ }^{1}$ Escola Paulista de Medicina, Universidade Federal de São Paulo. R. Botucatu 862, Vila Clementino. 04023-062 São Paulo SP Brasil. taticastroamato@gmail.com
Abstract The scope of this article is to describe and estimate the odds of problems among adolescents who reported binge drinking compared to those who reported alcohol consumption without binge drinking. The sample included 10,666 fourteen to eighteen-year-old public and private high school students in the 27 Brazilian state capitals who reported alcohol consumption at least once in the 12 months prior to the survey The odds of problems arising were estimated by ordinal logistic regression. Half of the students reported binge drinking and $33.1 \%$ admitted to at least one problem as a result. Binge drinking in the year $(a O R=4.7 ; C I: 3.9-5.7)$ and month $(a O R=4.4$; CI:3.6-5.4) was associated with greater odds of reported problems. The most likely problems were: going to school or work drunk (aOR:6.5; CI:3.611.9); having sex without a condom (aOR:5.0; CI:3.7-6.8); and getting into a fight (aOR:4.5; CI:3.2-6.3). Adolescents who go binge drinking are more exposed to alcohol-related problems than those who report lower alcohol consumption. It is suggested that alcohol education/prevention programs should consider the most prevalent problems and risk behaviors, thereby broadening the discussion on consumption of alcohol versus non-consumption of alcohol.

Key words Adolescents, Binge drinking, Alcohol, Risk behaviors
Resumo O objetivo deste artigo é descrever e estimar a chance de problemas entre adolescentes que referiram consumo binge drinking comparados aos que referiram ter consumido álcool sem essa característica. Participaram 10.666 estudantes de ensino médio de escolas públicas e privadas das 27 capitais brasileiras ( 14 a 18 anos) que declararam uso de álcool ao menos uma vez nos 12 meses anteriores à pesquisa. A chance de ocorrerem problemas devido ao consumo foi estimada por regressão logística ordinal. Metade dos estudantes relatou binge drinking e 33,1\% relatou ter vivido pelo menos um problema em decorrência do seu uso. $O$ binge drinking no ano $(a O R=4,7$; IC:3,9-5,7) e mês ( $a O R=4,4$; IC: 3,6-5,4) foi associado à maior chance de relato de problemas. Os problemas com maior chance de ocorrer foram: ir para a escola ou trabalho embriagado $(a O R=6,5$; IC:3,6-11,9); prática de relação sexual sem preservativo $(a O R=5,0 ; I C: 3,7-6,8)$; e entrar em brigas $(a O R=4,5 ; I C: 3,2-6,3)$. Adolescentes que praticam binge drinking estão mais expostos a problemas decorrentes de seu consumo do que aqueles que beberam em menor quantidade. Sugere-se que programas preventivos sobre álcool considerem os problemas mais prevalentes e padrões de uso mais arriscados, ampliando a dicotomia consumo versus não consumo.

Palavras-chave Adolescentes, Binge, Beber pesado episódico, Álcool, Comportamentos de risco 


\section{Introdução}

Apesar de ampla aceitação social, o consumo indevido de bebidas alcoólicas acarreta problemas consideráveis para o indivíduo e a sociedade, a curto e longo prazos ${ }^{1}$. Dados do Relatório Mundial sobre Drogas da United Nations Office on Drugs and Crime (UNODC) apontam as bebidas alcoólicas como a substância psicoativa mais consumida no mundo, sendo a população de adolescentes destacada como em particular risco para consequências do uso de álcool ${ }^{2,3}$.

Ao longo de anos os estudos sobre consumo de álcool na adolescência foram focados em padrões das duas extremidades de consumo, a experimentação e a dependência, alertando que o início precoce do uso de álcool é um dos fatores associados ao desenvolvimento do abuso e dependência ${ }^{4,5}$. Apesar disso, a maioria dos adolescentes que experimentam bebidas alcoólicas não desenvolvem consumo abusivo ou dependência. Em um estudo nacional com adolescentes americanos com idade entre 13 e 14 anos, dentre os $42 \%$ que relataram ter consumido álcool, 23\% evoluíram para uso regular de álcool e 13\% foi classificado com consumo abusivo com ou sem dependência de álcool ${ }^{6}$. Dessa forma, outros padrões de consumo têm sido estudados a fim de melhor compreender suas associações a prejuízos relacionados ao uso de álcool.

Atualmente um padrão de consumo de álcool amplamente explorado em estudos entre adolescentes é o binge drinking, definido como o consumo de cinco ou mais doses em uma mesma ocasião ${ }^{7}$. Este padrão de consumo é mais prevalente entre jovens de 14 a 34 anos e tende a diminuir após essa idade ${ }^{8,9}$. Entre estudantes norte-americanos, estudos demonstram um declínio na prevalência do binge drinking entre os anos de 1980 e 2010 - de $41 \%$ para $23 \%{ }^{10}$. Por sua vez, dados do European School Survey Project on Alcohol and Other Drugs (ESPAD) apontaram um aumento de 29\% em 1995 para 41\% em 2007 no binge drinking durante o último mês. Entretanto, dados do ESPAD de 2011 também mostraram uma diminuição para 38\% na prevalência deste padrão de consumo ${ }^{11}$. No Brasil, entre adolescentes de 14 a 18 anos inseridos na rede de ensino pública e privada das 27 capitais, a prevalência de binge drinking foi de $31,6 \%$ no ano e 20,9\% no mês anterior à pesquisa ${ }^{12}$. Além disso, tal padrão de consumo tem se mostrado mais prevalente em estudantes de classes econômicas mais altas, diferente do que ocorre em outros países ${ }^{12,13}$.

A adolescência é um importante período de transformações do ciclo vital, no qual o consumo de álcool expõe o adolescente a uma série de riscos sociais e à saúde ${ }^{14}$. Em amplo levantamento com estudantes norte-americanos observouse que aqueles que relataram a prática do binge drinking foram mais associados a diversos comportamentos de risco comparados a bebedores que não praticaram o binge drinking ${ }^{15}$. Dentre os comportamentos estão: pegar carona com motorista que bebeu, usar outras drogas, ser sexualmente ativo, ser vítima de violência por parceiro íntimo e tentar suicídio. Além disso, tal estudo também indicou que quanto maior a frequência do binge drinking, maior a chance de envolvimento nesses comportamentos de risco. Outros comportamentos também já foram estudados com jovens de outros países como: entrar em brigas e apresentar outros comportamentos violentos $^{16,17}$, ter relações sexuais forçadas entre meninas ${ }^{18}$, sexo sem proteção ${ }^{18}$, morte em acidentes automobilísticos ${ }^{19}$. Entretanto, ainda há escassez de estudos brasileiros de abrangência nacional.

Muitas inferências são realizadas sobre as consequências do consumo de álcool por adolescentes no Brasil ${ }^{8,20}$. No entanto, o principal estudo com estudantes de abrangência nacional PENSE (Pesquisa Nacional da Saúde do Escolar) cobre uma faixa etária muito restrita (alunos de $9^{\circ}$ ano do ensino fundamental) ${ }^{21}$. Além disso, existem poucos estudos que comparam diferentes padrões de consumo para adolescentes dificultando uma discussão ponderada sobre os níveis de risco com os quais os jovens se envolvem. Assim, as evidências sobre consumo de álcool na adolescência ficam mais restritas a consumir ou não consumir. Além disso, ainda não foram mensurados quais os comportamentos de risco e consequências são mais prováveis de acontecer devido ao consumo de álcool, a partir de amostras nacionais de estudantes do ensino médio que incluam escolas públicas e privadas. O presente artigo pretende avançar no conhecimento sobre os riscos relacionados a diferentes padrões de uso de álcool, tendo como objetivo descrever e estimar a chance de problemas entre adolescentes que referem binge drinking comparados aos que referem ter consumido álcool sem binge drinking, em uma amostra de estudantes de ensino médio de escolas públicas e particulares das 27 capitais brasileiras. 


\section{Método}

\section{Participantes}

Este estudo é parte do VI Levantamento Nacional sobre o Consumo de Drogas Psicotrópicas entre Estudantes do Ensino Fundamental e Médio das Redes Pública e Privada de Ensino nas 27 Capitais Brasileiras - 2010, coordenado pelo CEBRID (Centro Brasileiro de Informações Sobre Drogas ${ }^{22}$. A amostra dos estudantes pesquisados é representativa de alunos de escolas públicas e privadas das 27 capitais brasileiras. Foram selecionadas amostras independentes de escolas públicas e privadas a partir da lista do INEP (Instituto Nacional de Estudos e Pesquisas Educacionais Anísio Teixeira), onde constam todas as escolas do Brasil. Para cada tipo de escola (pública e privada), foram criados três estratos: escolas com ensino médio e fundamental, escolas apenas com ensino médio e escolas apenas com ensino fundamental. O número de escolas sorteadas em cada estrato foi proporcional ao número de alunos dos estratos. Após o sorteio das turmas participantes, todos os alunos da classe foram convidados a participar da pesquisa.

O processo de amostragem chegou a um total de 923 escolas (545 públicas e 378 privadas). A amostragem completa pode ser consultada no relatório da pesquisa e em artigos científicos já publicados $^{12,22,23}$.

Para as análises deste artigo foram incluídos 10.666 alunos de ensino médio (14-18 anos), que declararam uso de álcool ao menos uma vez nos 12 meses anteriores à aplicação do questionário. Tal recorte foi realizado para atender aos objetivos deste estudo.

\section{Procedimentos para a coleta de dados}

O projeto passou pela aprovação do Comitê de Ética da Universidade Federal de São Paulo. O termo de consentimento foi assinado por um representante responsável por cada escola (diretor ou coordenador).

Foi aplicado um questionário fechado e de autopreenchimento, coletivamente em sala de aula, sem a presença do professor, após explicação do projeto. Para garantia de anonimato, os questionários não foram identificados e foram colocados pelos alunos em envelope único por sala. Os estudantes tiveram a liberdade de não responder ao questionário e devolvê-lo em branco.

\section{Instrumentos}

\section{Padrões de uso de álcool}

Para avaliar o uso de bebidas alcoólicas, foram utilizadas perguntas baseadas em instrumento proposto pela Organização Mundial da Saúde ${ }^{24}$ e no questionário da European School Survey Project on Alcohol and Other Drugs (ESPAD $)^{25}$.

O uso no ano de álcool foi avaliado através da pergunta: "De um ano para cá, ou seja, nos últimos 12 meses, você tomou alguma bebida alcoólica?" sendo as opções de resposta sim ou não.

O padrão de consumo beber pesado episódico foi definido como o consumo de cinco ou mais doses de álcool em uma única ocasião ${ }^{7}$, sendo considerado uma dose padrão de $14 \mathrm{~g}$ de álcool, o equivalente a $350 \mathrm{ml}$ cerveja, $140 \mathrm{ml}$ de vinho ou $40 \mathrm{ml}$ de destilado. Em relação ao binge drinking foram incluídas duas questões: 1) "De um ano para cá, ou seja, nos últimos 12 meses, você tomou 5 doses ou mais de bebida alcoólica numa mesma ocasião?", sendo as opções de resposta sim, não e não sei; 2) "De um mês para cá, ou seja, nos últimos 30 dias, quantas vezes você tomou 5 doses ou mais de bebida alcoólica numa mesma ocasião?" sendo as opções de resposta: nenhuma vez, 1 vez, 2 vezes, 3 a 5 vezes, 6 a 9 vezes, 10 ou mais vezes e não lembro.

Para as análises estatísticas os participantes foram categorizados em dois grupos: aqueles que relataram o consumo de álcool no último ano, mas sem binge drinking, e os que consumiram álcool no último ano, incluindo no padrão de binge drinking.

\section{Problemas relacionados ao consumo de álcool}

Para avaliar os problemas relacionados ao consumo de álcool nos últimos 12 meses foram utilizados os dados sobre a frequência (nenhuma vez, 1-2 vezes, 3-5 vezes, 6 vezes ou mais) das seguintes situações associadas ao uso de álcool:

a) sofreu acidentes ou ferimentos

b) não foi capaz de fazer suas tarefas escolares ou estudar para uma prova

c) entrou em briga com parentes, amigos ou estranhos

d) foi para o trabalho ou para a escola "alto" ou embriagado

e) perdeu um dia (ou parte de um dia) de escola ou trabalho

f) envolveu-se em relações sexuais sem preservativo (camisinha)

g) foi vítima de roubo ou furto 
h) foi hospitalizado ou teve que ir a um pronto-socorro (PS)

Os tópicos acima (b, c, d, e) foram extraídos da RAPI (Rutgers Alcohol Problem Index) ${ }^{26} \mathrm{e}$ os demais ( $\mathrm{a}, \mathrm{f}, \mathrm{g}, \mathrm{h}$ ) do questionário do ESPAD (European School Survey Project on Alcohol and Other Drugs $)^{25}$.

\section{Crítica e análise dos dados}

A fase de crítica aos dados envolveu duas subfases independentes e sucessivas ${ }^{27}: 1$ ) crítica quantitativa: foi realizada conferência de $10 \%$ dos questionários para detectar e corrigir erros de digitação através de respostas com valores impossíveis (por exemplo: um aluno com 200 anos); 2) crítica qualitativa: o fato de cada uma das questões ser composta por vários itens permitiu a realização de testes de coerência interna como, por exemplo, a identificação de incoerência se o aluno responder não para uso de álcool na vida e sim para o uso no ano. Nesses casos a resposta incoerente foi corrigida.

Inicialmente conduziu-se a análise descritiva para caracterização da amostra (idade, gênero e tipo de escola) e dos problemas relacionados ao consumo de álcool, de acordo com os padrões de uso de álcool avaliados.

As hipóteses de associação entre o binge drinking e os comportamentos de risco consequentes foram verificadas a partir de testes qui quadrado e regressões logísticas. Modelos de regressão logística ordinal foram utilizados para estudar a associação entre os comportamentos de risco e frequências do binge drinking no mês. Os modelos foram ajustados pelas variáveis sociodemográficas (idade, gênero e tipo de escola). Os dados foram analisados no software estatístico STATA $11^{28}$. O nível mínimo de significância adotado foi de $99 \%$.

\section{Resultados}

A amostra do presente trabalho possui idade média de 16,0 anos ( $\mathrm{EP}=0,04), 55,4 \%$ de mulheres (IC99\%: 53,6-57,1) e 75,2\% (IC99\%: 70,3-79,5) de estudantes da rede pública de ensino.

Entre os estudantes que consumiram álcool no último ano, $50 \%$ relatou ter feito binge drinking (IC99\%: 47,4-52,6). O consumo binge no último mês foi relatado por 32,6\% (IC99\%: 30,0-35,2) dos estudantes. Um terço dos estudantes $(33,1 \%$, IC99\%: 31,2-35,0) relatou ter vivido pelo menos um problema em decorrência do seu uso de álco- ol nos últimos 12 meses. Na amostra geral, sem considerar o padrão de consumo, os problemas mais prevalentes foram: entrar em brigas $(11,5 \%$, IC99\%: 10,3-12,9), perder um dia de escola ou trabalho (11,0\%, IC99\%: 9,8-12,3), prática de relação sexual sem preservativo (10,6\%, IC99\%: 9,3-12,1) e deixar de fazer tarefas escolares ou estudar para prova (10,6\%, IC99\%: 9,2-12,7).

Comparados a estudantes que beberam no último ano, mas não praticaram binge drinking, alunos que relataram ter feito este tipo de consumo foram mais associados a todos comportamentos de risco estudados. A prevalência de brigas entre aqueles que relataram binge no ano foi de $18,7 \%$ (IC99\%: 16,4-21,3), perder um dia de escola ou trabalho 17,2\% (IC99\%: 14,9-19,6), prática de relação sexual sem preservativo $17,7 \%$ $(15,3-20,4)$ e deixar de fazer tarefas escolares ou estudar para prova $16,3 \%$ (IC99\%: 14,0-18,8) (Tabela 1). Em contrapartida, entre aqueles que beberam, mas não praticaram binge 4,6\% (IC99\%: 3,5-5,8) relataram ter entrado em brigas, 5\% (IC99\%: 4,1-6,2) perderam um dia de escola ou trabalho, 3,6\% (IC99\%: 2,8-4,5) praticou relação sexual sem preservativo e 5\% (IC99\%: 4,0-6,2) deixou de fazer tarefas escolares ou estudar para prova (Tabela 1).

A Tabela 2 apresenta as associações entre a frequência de binge drinking no último mês, gênero, tipo de escola e os comportamentos de risco. Maiores frequências de binge drinking no último mês foram associadas a maior prevalência de problemas. Para os adolescentes que relataram prática de binge seis vezes ou mais no mês a prevalência de brigas chegou a 36,2\% (IC99\%: $28,3-45,0$ ), enquanto que para quem não praticou binge no mês a prevalência de brigas foi 6,2\% (IC99\%: 5,1-7,4). Apenas entre aqueles que relataram ter sido hospitalizado ou foram para pronto socorro, a maior prevalência foi observada entre os que relataram praticar binge drinking entre 3 e 5 vezes no mês (7,6\%, IC99\%: 4,8-11,9, $\mathrm{p}<0,0001)$ ao invés de 6 ou mais vezes $(6,6 \%$, IC99\%: 3,8-11,4, p < 0,0001) (Tabela 2).

Os modelos de regressão logística demonstraram que quanto maior a idade, maior a chance de se relatar binge drinking no ano $(\mathrm{aOR}=1,4$ : IC99\%: 1,3-1,5) e mês (aOR = 1,4: IC99\%: 1,31,6). Da mesma forma, estudar em escola privada foi associado à maior chance de binge drinking no ano $(\mathrm{aOR}=1,4$ : IC:1,1-1,7) e no mês $(\mathrm{aOR}=1,4$ : IC:1,1-1,7). Mulheres foram menos associadas a chance de praticar binge drinking no ano $(\mathrm{aOR}=$ 0,6: IC99\%: 0,5-0,8) e mês (aOR = 0,7: IC99\%: $0,6-0,8)$. 
Tabela 1. Variáveis sociodemográficas e problemas associados ao consumo de álcool no ano com e sem a prática do binge drinking $(\mathrm{N}=10.666)$.

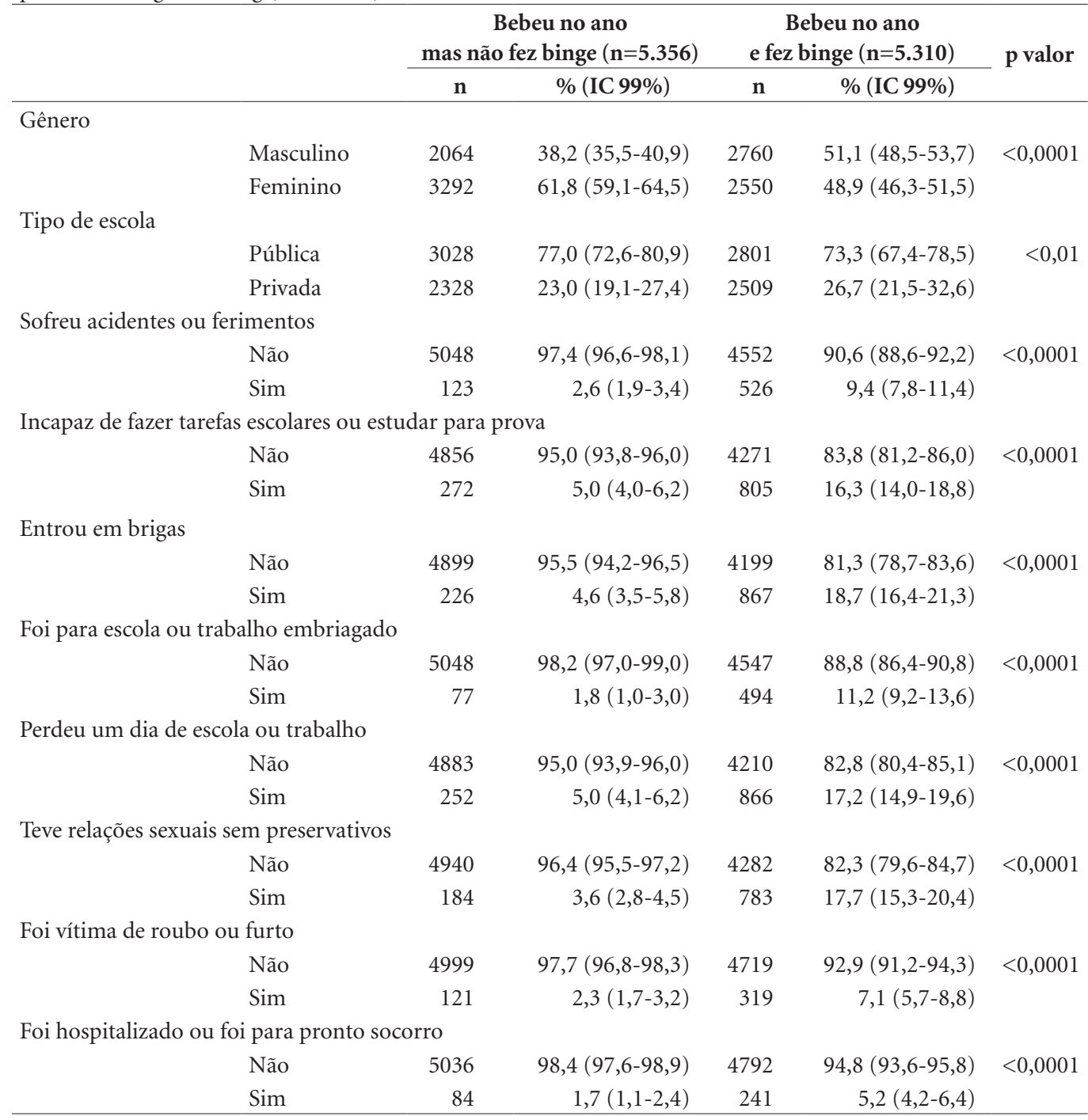

Variações do tamanho da amostra em cada variável de interesse são decorrentes da presença de missings.

A prática do binge drinking no ano e mês foi associada à maior chance de relato de problemas $(\mathrm{aOR}=4,7: \mathrm{IC} 99 \%: 3,9-5,7$ e aOR $=4,4:$ IC99\%: $3,6-5,4$, respectivamente). A Tabela 3 apresenta a comparação entre estudantes que beberam no último ano, mas não praticaram binge drinking e estudantes que praticaram o binge drinking. A prática do uso de álcool no padrão binge drinking foi mais associada à chance de relato de todos os problemas estudados. Entre os problemas com maior chance de ocorrer observou-se: ir para a escola ou trabalho embriagado (aOR = 6,5: IC99\%: 3,611,9); prática de relação sexual sem preservativo
$(\mathrm{aOR}=5,0$ : IC99\%: 3,7-6,8); e entrar em brigas (aOR = 4,5: IC99\%: 3,2-6,3) (Tabela 3).

Modelos de regressão logística ordinal permitiram observar um aumento do relato de problemas relacionados ao álcool na medida em que aumentam as frequências de relatos de binge drinking no mês (1-2 vezes; $3-5$ vezes e 6 ou mais vezes). As chances de se relatar problemas aumentam à medida que aumenta a frequência do consumo binge drinking no mês. A única exceção foi em relação a ser hospitalizado ou ir para o pronto socorro, no qual usuários com frequência intermediária ( 3 a 5 vezes por mês) tiveram 
Tabela 2. Variáveis sociodemográficas e problemas associados à frequência do binge drinking no último mês $(\mathrm{N}=10.333)$.

\begin{tabular}{|c|c|c|c|c|c|c|c|c|c|}
\hline & \multicolumn{8}{|c|}{ Bebeu e praticou o beber pesado episódico } & \multirow{3}{*}{ p valor } \\
\hline & \multicolumn{2}{|c|}{$\begin{array}{l}\text { Nenhuma vez } \\
(n=6.986)\end{array}$} & \multicolumn{2}{|c|}{$1-2$ vezes $(n=1.972)$} & \multicolumn{2}{|c|}{$3-5$ vezes $(n=839)$} & \multicolumn{2}{|c|}{$\begin{array}{l}6 \text { ou mais vezes } \\
(n=536)\end{array}$} & \\
\hline & $\mathbf{n}$ & $\%($ IC 99\%) & $\mathbf{n}$ & $\%($ IC 99\%) & $\mathbf{n}$ & $\%($ IC 99\%) & $\mathbf{n}$ & $\%($ IC 99\%) & \\
\hline \multicolumn{10}{|l|}{ Gênero } \\
\hline Masculino & 2865 & $40,7(38,5-42,9)$ & 966 & $47,9(43,5-52,4)$ & 471 & $55,0(48,2-61,6)$ & 348 & $57,9(49,7-65,7)$ & $<0,0001$ \\
\hline Feminino & 4121 & $59,3(57,1-61,5)$ & 1006 & $52,1(47,7-54,5)$ & 368 & $45,0(38,4-51,8)$ & 188 & $42,1(34,3-50,3)$ & \\
\hline \multicolumn{10}{|l|}{ Tipo de escola } \\
\hline Pública & 3914 & $76,1(71,1-80,5)$ & 971 & $70,4(63,1-76,9)$ & 407 & $70,5(63,1-76,9)$ & 334 & $82,2(75,7-87,2)$ & $<0,0001$ \\
\hline Privada & 3072 & $23,9(19,5-28,9)$ & 1001 & $29,6(23,1-36,1)$ & 432 & $29,6(23,1-36,9)$ & 202 & $17,8(12,8-24,3)$ & \\
\hline \multicolumn{10}{|c|}{ Sofreu acidentes ou ferimentos } \\
\hline Não & 6539 & $96,8(95,8-97,6)$ & 1719 & $92,3(90,0-94,2)$ & 676 & $87,2(82,2-91,0)$ & 393 & $81,9(75,1-87,3)$ & $<0,0001$ \\
\hline Sim & 207 & $3,2(2,4-4,2)$ & 170 & $7,7(5,8-10,0)$ & 123 & $12,8(9,0-17,9)$ & 108 & $18,1(12,8-24,9)$ & \\
\hline \multicolumn{10}{|c|}{ Incapaz de fazer tarefas escolares ou estudar para prova } \\
\hline Não & 6274 & $93,7(92,4-94,7)$ & 1630 & $86,6(83,1-89,4)$ & 623 & $77,2(71,5-82,1)$ & 366 & $74,1(35,9-80,9)$ & $<0,0001$ \\
\hline Sim & 424 & $6,3(5,3-7,6)$ & 262 & $13,4(10,6-16,9)$ & 178 & $22,8(17,9-28,5)$ & 135 & $25,9(19,1-34,1)$ & \\
\hline \multicolumn{10}{|l|}{ Entrou em brigas } \\
\hline Não & 6308 & $93,8(92,6-94,9)$ & 1611 & $83,9(80,3-87,0)$ & 619 & $76,8(69,8-82,6)$ & 334 & $63,8(55,0-72,8)$ & $<0,0001$ \\
\hline Sim & 388 & $6,2(5,1-7,4)$ & 278 & $16,1(13,1-19,7)$ & 179 & $23,2(17,4-30,2)$ & 166 & $36,2(28,3-45,0)$ & \\
\hline \multicolumn{10}{|c|}{ Foi para escola ou trabalho embriagado } \\
\hline Não & 6552 & $97,4(96,2-98,2)$ & 1743 & $92,2(89,6-94,1)$ & 668 & $83,0(76,0-88,2)$ & 374 & $72,8(64,1-80,0)$ & $<0,0001$ \\
\hline Sim & 142 & $2,6(1,8-3,8)$ & 129 & $7,9(5,9-10,4)$ & 127 & $17,0(11,8-24,0)$ & 124 & $27,2(20,0-35,9)$ & \\
\hline \multicolumn{10}{|c|}{ Perdeu um dia de escola ou trabalho } \\
\hline Não & 6296 & $94,1(93,1-95,0)$ & 1599 & $84,5(80,9-87,6)$ & 614 & $75,7(69,2-81,2)$ & 344 & $68,7(58,6-77,2)$ & $<0,0001$ \\
\hline Sim & 417 & $5,9(5,0-6,9)$ & 297 & $15,5(12,4-19,1)$ & 181 & $24,3(18,8-30,8)$ & 158 & $31,3(22,8-41,4)$ & \\
\hline \multicolumn{10}{|c|}{ Teve relações sexuais sem preservativos } \\
\hline Não & 6371 & $94,8(93,5-95,9)$ & 1663 & $85,6(81,8-88,8)$ & 616 & $76,6(70,8-81,6)$ & 337 & $64,4(56,2-71,8)$ & $<0,0001$ \\
\hline Sim & 322 & $5,2(4,1-6,5)$ & 226 & $1,4(11,3-18,3)$ & 183 & $23,4(18,4-29,2)$ & 159 & $35,7(23,2-43,8)$ & \\
\hline \multicolumn{10}{|c|}{ Foi vítima de roubo ou furto } \\
\hline Não & 6499 & $97,1(96,2-97,9)$ & 1768 & $92,7(88,5-95,5)$ & 731 & $92,0(87,7-94,9)$ & 436 & $88,2(82,5-92,3)$ & $<0,0001$ \\
\hline Sim & 188 & $2,9(2,1-3,8)$ & 109 & $7,3(4,5-11,5)$ & 62 & $8,0(5,1-12,3)$ & 59 & $11,8(7,7-17,5)$ & \\
\hline \multicolumn{10}{|c|}{ Foi hospitalizado ou foi para pronto socorro } \\
\hline Não & 6558 & $98,0(97,2-98,5)$ & 1798 & $95,1(92,4-96,8)$ & 738 & $92,4(88,1-95,2)$ & 445 & $93,4(88,7-96,3)$ & $<0,0001$ \\
\hline Sim & 129 & $2,0(1,5-2,8)$ & 72 & $4,9(3,2-7,6)$ & 56 & $7,6(4,8-11,9)$ & 49 & $6,6(3,8-11,4)$ & \\
\hline
\end{tabular}

Variações do tamanho da amostra em cada variável de interesse são decorrentes da presença de missings.

maior chance de relatar tal comportamento $(\mathrm{aOR}=3,3:$ IC99\%: 1,8-6,4) (Tabela 3).

Quanto às variáveis de controle utilizadas neste estudo, houve diferenças quanto à idade, gênero e tipo de escola que devem ser ressaltadas. Quanto maior a idade do adolescente, maior a chance de praticar binge no mês $(\mathrm{aOR}=1,4$ : IC99\%: 1,3-1,5) e no ano e de relatar ao menos uma resposta de comportamento de risco tanto no mês quanto no ano ( $\mathrm{aOR}=1,2$ : IC99\%: 1,2$1,3)$. Adolescentes de escolas privadas, por exemplo, tiveram menor chance de fazer sexo sem proteção (aOR = 0,4: IC99\%: 0,3-0,6) e foram mais prováveis de se envolverem em acidentes no ano $(\mathrm{aOR}=1,5:$ IC99\%: 1,1-2,1). O gênero dos respondentes também teve relação com o padrão de consumo de álcool e com alguns problemas associados. Adolescentes do sexo feminino tiveram menor chance de praticar binge tanto no mês $(\mathrm{aOR}=0,7: \mathrm{IC} 99 \%: 0,5-0,8)$ quanto no ano (aOR = 0,6: IC99\%: 0,5-0,7) e também menor probabilidade de envolvimento em brigas $(\mathrm{aOR}=0,7$ : IC99\%: 0,5-0,9), bem como o relato de relação sexual sem proteção ( $\mathrm{aOR}=0,6$ : IC99\%: 0,4$0,8)$. As meninas também apresentaram menos chance de serem hospitalizadas por binge no mês ( $\mathrm{aOR}=0,5:$ IC99\%: 0,3-0,9), mas esta diferença não se manteve para a análise sobre binge no ano. 


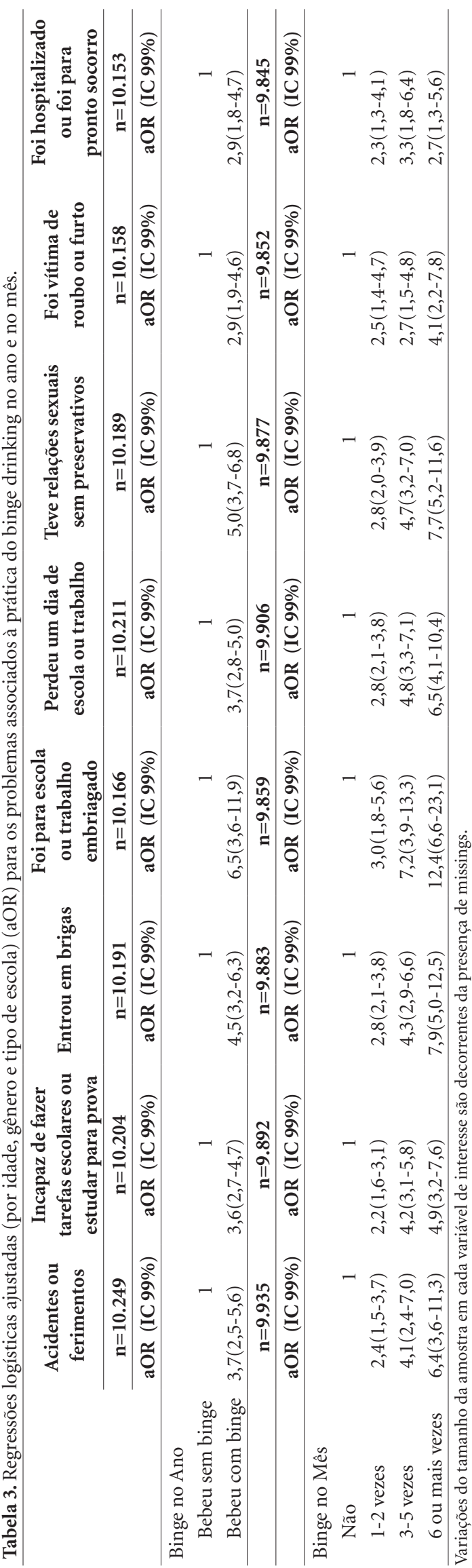

\section{Discussão}

Os dados apontaram que adolescentes que praticam binge drinking estão mais expostos a problemas decorrentes de seu consumo do que aqueles que beberam em menor quantidade por ocasião. Cerca de um terço dos jovens viveram pelo menos um dos problemas estudados em consequência do uso de álcool. Essa prevalência aumenta para os casos que referem padrão binge e quanto maior a frequência de binge, maiores as chances de consequências negativas. Os comportamentos mais relatados foram brigas, perdas de dias de escola ou trabalho, prática de relação sexual sem preservativo e deixar de fazer tarefas escolares ou estudar para prova. Foram encontradas ainda diferenças entre os tipos de escola e de gênero, tais dados estão de acordo com outros estudos na literatura internacional ${ }^{15,29-31}$.

O presente estudo constitui-se o primeiro a mensurar a prevalência de problemas associados ao consumo de álcool e com enfoque no padrão binge de consumo, entre estudantes de escolas públicas e privadas de todas as capitais brasileiras. A abrangência de todas as capitais nacionais na pesquisa, associada à coleta de dados em duas amostras estudantis (redes pública e privada), fornecem a prevalência desses comportamentos em estudantes de diferentes estratos socioeconômicos, permitindo o estabelecimento de prioridades educacionais na área. Os achados ganham relevância diante da considerável prevalência de estudantes brasileiros que referem consumo de álcool.

Além disso, ainda são poucas as pesquisas no Brasil que se dedicam a avaliar os riscos associados aos diferentes padrões de consumo de álcool entre os adolescentes. Geralmente as pesquisas comparam adolescentes que consomem álcool com aqueles que não consomem ${ }^{12,13}$. Os dados do presente estudo preenchem a lacuna do detalhamento dos riscos de distintos padrões de consumo, mostrando que beber em maior quantidade traz mais riscos do que beber poucas doses. Nossos dados mostraram que adolescentes que praticaram binge seis vezes no mês chegaram a estar oito vezes mais propensos a se envolverem em brigas, o comportamento mais prevalente em decorrência do consumo. No entanto, mesmo a baixa frequência de binge aumentou em quatro vezes a chance de brigar.

Independente da motivação que leva o jovem ao uso de álcool, nossos dados revelaram que uma proporção significativa de estudantes brasileiros se expõe a comportamentos de risco 
à saúde associados ao consumo agudo de bebida, fenômeno que também tem sido observado no cenário internacional ${ }^{15,32}$. Se envolver em brigas e a prática de relação sexual sem preservativo particularmente podem promover consequências significativamente impactantes à saúde e vida do adolescente, tanto a curto ou longo prazo.

As brigas e práticas de relação sexual sem preservativo estão associados ao consumo agudo, o que o torna um dos focos importantes para os programas preventivos. A adolescência configurase um período de início de consumo, sendo o uso crônico um padrão mais comum em outras faixas etárias ${ }^{33}$. Estudos brasileiros e de outros países já mostraram que a maior preocupação reportada pelos jovens está mais relacionada às consequências do consumo agudo de álcool, provavelmente decorrentes do binge drinking ${ }^{34,35}$. Dessa forma, apesar da dependência do álcool ser assunto de extrema importância em programas preventivos, a ênfase maior em consequências como brigas e ter relações sexuais sem proteção parecem fazer mais sentido à experiência de jovens nessa faixa etária, portanto merecem maior destaque em programas preventivos. Programas preventivos que adotam discurso mais próximo à realidade dos jovens tendem a aumentar a adesão dos jovens às atividades e constroem junto aos mesmos uma visão mais realista e menos amedrontadora sobre o consumo de álcool ${ }^{36-38}$. Estes resultados poderão ser usados para subsidiar discussão em políticas públicas e poderão ser explorados na criação de ações preventivas voltadas para redução dos riscos reais associados ao consumo de bebidas alcoólicas por adolescentes em idade escolar.

As ações de redução de riscos ao consumo de álcool entre adolescentes são relativamente novas no ambiente escolar e, como demonstram os dados do presente artigo, existe demanda na escola para adoção deste modelo de intervenção. Alguns países têm adotado a redução de riscos em programas preventivos para estudantes, com significativa diminuição das consequências decorrentes do consumo, bem como no próprio consumo ${ }^{39,40}$. Para muitas estratégias preventivas atuais o não consumo de álcool é colocado como a única estratégia válida para diminuir suas consequências, sendo que os dados sugerem que possivelmente a redução de consumo em frequência e quantidade também podem diminuir os riscos entre adolescentes. Devido à expectativa de não consumo pelos adolescentes, outros padrões de uso que são praticados por eles acabam não sendo discutidos em programas preventivos que adotam uma abordagem de abstinência. A amplia- ção dessas estratégias nos programas preventivos pode aumentar a adesão dos jovens por serem úteis à realidade da maioria que já bebeu. Além disso, podem aumentar o repertório dos jovens para se protegerem.

Nossos dados mostram que as consequências do consumo de álcool tendem a ser diferentes entre alunos de escolas públicas e privadas. No Brasil alunos de escolas particulares possuem maior poder aquisitivo e por isso tem maior chance de irem de carro a festas mais longe de casa, o que provavelmente aumenta a chance de se envolverem em acidentes, assemelhando-se a resultados encontrados na literatura ${ }^{41}$. Porém, esses adolescentes de escolas particulares também se mostraram menos prováveis de praticarem sexo sem proteção. Os dados não permitem inferir o motivo que leva estes alunos a se protegerem mais sobre o uso de preservativo. Dessa forma, sugere-se que potenciais fatores de proteção sejam investigados em estudos futuros, no sentido de buscar subsídios para ações preventivas relacionadas a doenças sexualmente transmissíveis e gravidez na adolescência. Estudos internacionais costumam encontrar diferenças de consumo de álcool e de comportamentos associados nas classes econômicas mais baixas e mais altas. No entanto, contrário ao que foi encontrado, estudos em outros países evidenciam maior risco em populações com menor poder aquisitivo ${ }^{41-43}$.

É consenso na literatura a diferença de gênero em relação aos efeitos do álcool no organismo e no comportamento do adolescente ${ }^{44}$. Mulheres têm menos massa muscular e diferenças de metabolismo do álcool por isso são mais suscetíveis às consequências fisiológicas do uso de bebidas ${ }^{45}$. Adolescentes do sexo masculino, por sua vez, apresentam maiores problemas externos, principalmente de ordem comportamental ${ }^{44}$, como os que apareceram em nosso estudo. Meninos apresentaram maior chance de se envolverem em brigas, serem hospitalizados e praticarem sexo sem proteção. O padrão de consumo entre meninos e meninas também se mostrou diferente, como já demonstra a literatura científica. Meninos bebem, geralmente em maior quantidade e com mais frequência do que as meninas, no entanto, quando bebem, é comum elas praticarem binge $e^{46}$. A importância em conhecer os principais riscos relacionados a cada gênero pode ser um caminho para trabalhar medidas preventivas mais realistas e efetivas, potencializar possíveis fatores de proteção e subsidiar serviços de atendimento ${ }^{45}$.

Algumas limitações devem ser levadas em consideração na discussão dos resultados obti- 
dos no presente estudo. Em primeiro lugar, as informações utilizadas nas análises apresentadas basearam-se em dados de autorrelato. O uso de questionários anônimos, de preenchimento voluntário e a garantia de confidencialidade das informações foram procedimentos adotados com o intuito de minimizar essa limitação.

Em segundo lugar, apesar da abrangência nacional do presente estudo, o fato de este incluir apenas escolas localizadas nas capitais dos estados brasileiros apresenta-se como uma limitação importante, uma vez que os resultados encontrados podem não corresponder à realidade de escolas localizadas em outros municípios que não os pesquisados. É importante considerar também que todas as informações deste estudo correspondem à fração de adolescentes que frequentam o Ensino Médio no Brasil, nas capitais brasileiras. Portanto, os dados obtidos não po- dem ser expandidos para a realidade de adolescentes que, embora tenham a idade da amostra escolhida para esta pesquisa, não frequentam a escola. Outro fator importante é que a definição de binge drinking utilizada neste estudo foi de 5 doses para ambos os sexos, sendo assim, o dado para meninas pode estar subestimado, se comparado a estudos que utilizam a medidas de 4 doses para mulheres ${ }^{15,47}$.

Apesar das limitações, o estudo permite concluir que existem padrões de consumo de álcool na adolescência que podem levar a menos ou mais problemas. Os dados sugerem a necessidade de ações de prevenção que avancem para além da discussão dicotômica sobre consumir ou não consumir existente na maioria dos programas preventivos, bem como incluam estratégias focadas em minimizar as consequências dos padrões mais arriscados.

\section{Colaboradores}

K Gomes e TC Amato trabalharam na concepção do artigo, análises estatísticas, escrita do artigo e na revisão da versão final. A Bedendo trabalhou nas análises estatísticas, escrita do artigo e revisão da versão final. EL Santos trabalhou na escrita do artigo e revisão da versão final. AR Noto trabalhou na concepção do artigo, discussão dos resultados e direcionamento das análises estatísticas, escrita do artigo e revisão final.

\section{Agradecimentos}

Agradecemos ao CEBRID (Centro Brasileiro de Informações sobre Drogas) pela concessão do banco de dados para análise. Os dados foram coletados para o VI Levantamento Nacional sobre o Consumo de Drogas Psicotrópicas entre Estudantes do Ensino Fundamental e Médio das Redes Pública e Privada de Ensino nas 27 Capitais Brasileiras - 2010, financiado pela Secretaria Nacional de Política sobre Drogas (SENAD). O artigo é também fruto do trabalho de iniciação científica de Karla Gomes, bolsista FAPESP. 


\section{Referências}

1. Galduróz J, Noto A. Uso pesado de álcool entre estudantes de $1^{\circ}$ e $2^{\circ}$ graus da rede pública de ensino em dez capitais brasileiras. J Bras Dep Quím 2000; 1(1):2532.

2. World Organization Health (WHO). Global strategy to reduce the harmful use of alcohol. Geneva: WHO; 2010.

3. Escritório das Nações Unidas sobre Drogas e Crime (UNODC). World Drug Report 2012. New York: UNODC; 2012.

4. Grant JD, Scherrer JF, Lynskey MT, Lyons MJ, Eisen SA, Tsuang MT, True WR, Bucholz KK. Adolescent alcohol use is a risk factor for adult alcohol and drug dependence: evidence from a twin design. Psychol Med 2006; 36(1):109-118.

5. Hingson RW, Heeren T, Winter MR. Age at drinking onset and alcohol dependence: age at onset, duration, and severity. Arch Pediatr Adolesc Med 2006; 160(7):739-746.

6. Swendsen J, Burstein M, Case B, Conway KP, Dierker L, He J, Merikangas KR. Use and abuse of alcohol and illicit drugs in US adolescents: results of the National Comorbidity Survey-Adolescent Supplement. Arch Gen Psychiatry 2012; 69(4):390-398.

7. Wechsler H, Nelson TF. Binge drinking and the American college student: what's five drinks? Psychol Addict Behav 2001; 15(4):287-291.

8. Silveira CM, Silveira CC, Silva JG, Silveira LM, Andrade AG, Andrade LHSG. Epidemiologia do beber pesado e beber pesado episódico no Brasil: uma revisão sistemática da literatura. Revista de Psiquiatria Clínica 2008; 35(Supl. 1):31-38.

9. Kuntsche E, Gmel G. Alcohol consumption in late adolescence and early adulthood--where is the problem? Swiss Med Wkly 2013; 143:w13826.

10. Johnston L, O’Malley P, Bachman J, Schulenberg J. Monitoring the future national results on drug use: 2012 overview, key findings on adolescent drug use. Ann Arbor: Institute for Social Research, The University of Michigan; 2013.

11. Hibell B, Guttormsson U, Ahlström S, Balakireva O, Bjarnason T, Kokkevi A, Kraus L. The 2011 ESPAD report. Substance use among students in 36 European Countries. Stockholm: Swedish Council for Information on Alcohol and Other Drugs (CAN); 2012.

12. Sanchez ZM, Locatelli DP, Noto AR, Martins SS. Binge drinking among Brazilian students: a gradient of association with socioeconomic status in five geo-economic regions. Drug Alcohol Depend 2013; 127(1-3):87-93.

13. Locatelli D, Sanchez Z, Opaleye E, Carlini C, Noto AR. Socioeconomic influences on alcohol use patterns among private school students in São Paulo. Rev Bras Psiquiatr 2012; 34(2):193-200.

14. Barroso T, Mendes A, Barbosa A. Análise do fenômeno do consumo de álcool em adolescentes: estudo realizado com adolescentes do $3^{\circ}$ ciclo de escolas públicas. Revista Latino-Americana de Enfermagem 2009; 17(3):347-353.
15. Miller JW, Naimi TS, Brewer RD, Jones SE. Binge drinking and associated health risk behaviors among high school students. Pediatrics 2007; 119(1):76-85.

16. Swahn MH, Simon TR, Hammig BJ, Guerrero JL. Alcohol-consumption behaviors and risk for physical fighting and injuries among adolescent drinkers. Addict Behav 2004; 29(5):959-963.

17. Swahn MH, Donovan JE. Correlates and predictors of violent behavior among adolescent drinkers. J Adolesc Health 2004; 34(6):480-492.

18. Howard DE, Wang MQ. Psychosocial correlates of U.S. adolescents who report a history of forced sexual intercourse. J Adolesc Health 2005; 36(5):372-379.

19. Yi H, Williams GD, Dufour MC. Surveillance Report\# 61: Trends in alcohol-related fatal traffic crashes, United States, 1977-2000. Rockville: NIAAA, Division of Biometry and Epidemiology, Alcohol Epidemiologic Data System; 2002.

20. Pechansky F, Szobot CM, Scivoletto S. Uso de álcool entre adolescentes: conceitos, características epidemiológicas e fatores etiopatogênicos. Rev Bras Psiquiatr 2004; 26(Supl. I):14-17.

21. Instituto Brasileiro de Geografia e Estatística (IBGE). Pesquisa Nacional de Saúde do Escolar, 2012. Rio de Janeiro: IBGE; 2013.

22. Carlini EA, Noto AR, Sanchez ZM, Carlini CMA, Locatelli DP, Ribeiro LA, Amato TC, Opaleye ES, Tondowski CS, Moura YG. VI Levantamento Nacional sobre o Consumo de Drogas Psicotrópicas entre Estudantes do Ensino Fundamental e Médio das Redes Pública e Privada de Ensino nas 27 Capitais Brasileiras - 2010. Brasília: SENAD; 2012.

23. Bedendo A, Opaleye ES, Andrade AL, Noto AR. Heavy episodic drinking and soccer practice among high school students in Brazil: the contextual aspects of this relationship. BMC Public Health 2013; 13:247.

24. Smart RG, Hughes DPH, Johnston LD. Methodology for students drug-use surveys. Geneva: WHO; 1980.

25. Hibell B, Guttormsson U, Ahlström S, Balakireva O, Bjarnason T, Kokkevi A, Kraus L. The 2007 ESPAD report: substance use among students in 35 European countries. Stockholm: Swedish Council for Information on Alcohol and Other Drugs (CAN); 2009.

26. White HR, Labouvie EW. Towards the assessment of adolescent problem drinking. J Stud Alcohol 1989; 50(1):30-37.

27. Carlini-Cotrim B, Barbosa MTS. Pesquisas epidemiológicas sobre uso de drogas entre estudantes: Um manual de orientações gerais. São Paulo: Centro Brasileiro de Informações sobre Drogas; 1993.

28. StataCorp. Stata Statistical Software: Release 11. College Station: StataCorp LP; 2009.

29. Arata CM, Stafford J, Tims MS. High school drinking and its consequences. Adolescence 2003; 38(151):567579 . 
30. Danielsson AK, Romelsjo A, Tengstrom A. Heavy episodic drinking in early adolescence: gender-specific risk and protective factors. Subst Use Misuse 2011; 46(5):633-643.

31. Danielsson AK, Wennberg P, Hibell B, Romelsjo A. Alcohol use, heavy episodic drinking and subsequent problems among adolescents in 23 European countries: does the prevention paradox apply? Addiction 2012; 107(1):71-80.

32. Siegel MB, Naimi TS, Cremeens JL, Nelson DE. Alcoholic beverage preferences and associated drinking patterns and risk behaviors among high school youth. Am J Prev Med 2011; 40(4):419-426.

33. Laranjeira R, Pinsky I, Zaleski M, Caetano R. I Levantamento Nacional sobre os padrões de consumo de álcool na população brasileira. Brasília: Unidade de Pesquisa em Álcool e Drogas (Uniad), Universidade Federal de São Paulo, Secretaria Nacional de Políticas Antidrogas (SENAD); 2007.

34. Gonçalves LP, Opaleye ES, Amato TC. Percepção de riscos e estratégias de redução de danos do uso de álcool por adolescentes: subsídios para intervenções além do "diga não". Brasília: SENAD, CIEE; 2014.

35. Farringdon FM, McBride N, Midford R. The fine line: students' perceptions of drinking, having fun and losing control. Youth Studies Australia 2000; 19(3):32-38.

36. Gormana DMC, Conde E. The Making of Evidence-based Practice: The Case of Project ALERT. Child Youth Serv Rev 2010; 32(2):214-222.

37. McBride N. The evidence base for school drug education interventions. In: Stockwell T, Gruenewald P, Toumbourou J, Loxley W, editors. Preventing Harmful Substance Use: The evidence base for policy and practice UK. London: Wiley; 2005. p. 101-112.

38. Gosin MN, Dustman PA, Drapeau AE, Harthun ML. Participatory Action Research: creating an effective prevention curriculum for adolescents in the Southwestern US. Health Educ Res 2003; 18(3):363-379.

39. McBride N, Farringdon F, Midford R, Meuleners L, Phillips M. Harm minimization in school drug education: final results of the School Health and Alcohol Harm Reduction Project (SHAHRP). Addiction 2004; 99(3):278-291.

40. Mckay MT, Mcbride NT, Sumnall HR, Cole JC. Reducing the harm from adolescent alcohol consumption: results from an adapted version of SHAHRP in Northern Ireland. Journal of Substance Use. 2012; 17(2):98-121.

41. Yaogo A, Fombonne E, Lert F, Melchior M. Adolescent Repeated Alcohol Intoxication as a Predictor of Young Adulthood Alcohol Abuse: The Role of Socioeconomic Context. Subst Use Misuse 2015; 50(14):1795-804.

42. Brown M. Familial, social, and individual factors contributing to risk for adolescent substance use. J Addict 2013; 2013:579310.

43. Doom JR, Vanzomeren-Dohm AA, Simpson JA. Early unpredictability predicts increased adolescent externalizing behaviors and substance use: A life history perspective. Dev Psychopathol 2015; 28(4pt. 2):1505-1516.
44. Jun HJ, Sacco P, Bright CL, Camlin EA. Relations Among Internalizing and Externalizing Symptoms and Drinking Frequency During Adolescence. Subst Use Misuse 2015; 50(14):1814-1825.

45. Yue Y, Hong L, Guo L, Gao X, Deng J, Huang J, Huang $\mathrm{G}, \mathrm{Lu}$ C. Gender differences in the association between cigarette smoking, alcohol consumption and depressive symptoms: a cross-sectional study among Chinese adolescents. Sci Rep 2015; 5:17959.

46. Llerena S, Arias-Loste MT, Puente A, Cabezas J, Crespo J, Fabrega E. Binge drinking: Burden of liver disease and beyond. World J Hepatol 2015; 7(27):2703-2715.

47. Assanangkornchai S, Sam-Angsri N, Rerngpongpan S, Edwards JG. Anxiolytic and hypnotic drug misuse in Thailand: Findings from a national household survey. Drug Alcohol Rev 2010; 29(1):101-111.
Artigo apresentado em 29/12/2016

Aprovado em 26/04/2017

Versão final apresentada em 28/04/2017 